\title{
Acetaldehyde as the first hit of addictive behaviour
}

\author{
Angela Cavallaro, ${ }^{1}$ Gianluca Lavanco, ${ }^{1}$ Carla Cannizzaro, ${ }^{1}$ Anna Brancato, ${ }^{1}$ Danila Di Majo, ${ }^{2}$ \\ Marco Giammanco, ${ }^{2}$ Francesco Martines, ${ }^{3}$ Irene Miccichè, ${ }^{1}$ Fulvio Plescia ${ }^{1}$ \\ ${ }^{1}$ Department of Sciences for Health Promotion and Mother and Child Care Giuseppe D'Alessandro, \\ University of Palermo, Palermo; ${ }^{2}$ Department of Experimental Biomedicine and Clinical \\ Neurosciences, University of Palermo, Palermo; ${ }^{3}$ Department of Experimental Biomedicine and \\ Clinical Neurosciences, ENT Section, University of Palermo, Palermo, Italy
}

\begin{abstract}
Unhealthy alcohol use is common in the Western society, which puts risk of health consequences, causing multiple behavioural injuries. Increasing evidence focuses on acetaldehyde, the first metabolite of ethanol, as the mediator of the several behavioural actions of alcohol, including its rewarding and motivational effects. In particular, acetaldehyde induces dopamine release in the nucleus accumbens modulating primary alcohol rewarding effect, drug seeking, and relapse behaviour. Recent behavioural studies point at acetaldehyde as a drug of abuse since its oral self-administration is induced and maintained in an operant/conflict paradigm. These findings provide further evidence on the role played by the acetaldehyde as a mediator of the effects of alcohol and focus attention on this molecule to arrange a more effective strategy, aimed at the prevention and treatment of alcohol abuse. Thus, the aim of this review is to summarize latest results on the role of acetaldehyde as the mediator of ethanol-central effects focusing on its capacity to induce an addictive behaviour.
\end{abstract}

\section{Introduction}

Alcohol is regularly consumed, ranging from 8 to $10 \%$ of heavy alcohol use (an average of $\geq 14$ drinks per week in males and $\geq 7$ drinks

Correspondence: Fulvio Plescia, Department of Sciences for Health Promotion and Mother and Child Care "G. D'Alessandro", University of Palermo, via del Vespro 129, 90127 Palermo, Italy. Tel: +39.091.6555812. Email: fulvio.plescia@unipa.it

Key words: Acetaldehyde; Ethanol-related effects; Addictive behaviour.

Conflict of interest: the authors declare no potential conflict of interest.

Received for publication: 27 July 2016.

Revision received: 4 November 2016

Accepted for publication: 7 November 2016.

(C) Copyright A. Cavallaro et al., 2016

Licensee PAGEPress, Italy

Journal of Biological Research 2016; 89:6206

doi:10.4081/jbr.2016.6206

This article is distributed under the terms of the Creative Commons Attribution Noncommercial License (by-nc 4.0) which permits any noncommercial use, distribution, and reproduction in any medium, provided the original author(s) and source are credited. per week in females). ${ }^{1}$ Alcohol abuse represents a substantial and growing health problem in Western societies and it is the third risk factor for cardiovascular disease, cirrhosis of the liver and various cancers (WHO). The central effects of alcohol (ethanol, EtOH) are mediated by the interaction with different neurotransmitters, ionic channel, membrane proteins and receptors. ${ }^{2-4} \mathrm{EtOH}$ abuse is associated with impairments of intellectual functions, memory, verbal and non verbal learning, visual motor coordination, cognitive flexibility, executive functions, problem solving, decision making, perception and information processing speed. ${ }^{5-11}$ In the last years, EtOH dependence has been defined as a chronic relapsing and remitting disease, and it is well known that $\mathrm{EtOH}$ is able to influence emotional behaviour and cognition in humans in a dose-dependent manner, ${ }^{12}$ time and modality of administration. ${ }^{13}$ An important role in the neurobiology of ethanol addiction is played by acetaldehyde (ACD), ethanol first metabolite detected and analyzed by HPLC/MS, that allows a quantitative and qualitative analysis. ${ }^{14,15}$ It derives from ethanol oxidative metabolism, which occurs by peripheral alcohol dehydrogenase, and by central catalase and CYP2E1. ${ }^{16-18}$ High blood levels of ACD enter the brain, likely overwhelming the aldehyde dehydrogenase present in the blood-brain barrier. ${ }^{19}$ Muggironi and collegues showed experimental evidences on the involvement of ACD in the neurobiological mechanism supporting the motivational effects of EtOH. ${ }^{20}$ Several studies have recently focused on ACD as the mediator of rewarding and motivational properties of EtOH. ${ }^{21,22}$ In particular, it has been reported that ACD acts in the mesocorticolimbic system, affecting dopamine (DA) neurotransmission with an increase of the neuronal firing in the ventral tegmental area (VTA), stimulating DA release in the nucleus accumbens (NAc) shell. ${ }^{23-25}$ In the last decades, several research groups have focused their attention on the role of ACD as a compound with potentially addictive effects, suggesting that ACD itself mediates addiction and craving, ${ }^{8,26,27}$ thus playing a key role in the development of alcohol dependence. ${ }^{28,29}$

\section{Search methods}

The authors' search targeted evidence-based guidelines, evidencebased summaries, systematic reviews and recent experimental research on acetaldehyde formation in the brain and its role as the mediator of ethanol-central effects. The keywords used were $A C D$ or $A C D$ in the brain or dopaminergic pathway or EtOH-central effects or $A C D$ and EtOH-related addictive behaviour. Through this simple strategy, we identified more than 10000 using two primary sources to identify relevant information: PubMed and Scopus (last accessed via PubMed and Scopus on March, 2016). 


\section{Role of acetaldehyde in addictive behaviour}

The involvement of ACD as the primum movens of motivational and addictive properties of $\mathrm{EtOH}$ has been postulated by experiments conducted on manipulation of brain catalase activity. There is a positive correlation between brain catalase activity and natural propensity to drink EtOH in rodents, as shown by Amit and Aragon..$^{30}$ In agreement with past researches, these results confirmed that brain catalase activity and voluntary ethanol intake are unidirectionally and causally related, ${ }^{31}$ suggesting that brain catalase activity may be part of an enzymatic system, which controls the production and elimination of acetaldehyde in the brain. ${ }^{16,32}$ In particular, the administration of the irreversible inhibitor of catalase, 3 -amino-1,2,4-triazole is able to decrease $\mathrm{EtOH}$ intake, the induction of an ethanol-induced conditioning-taste aversion to saccharine, ${ }^{31}$ and to antagonize ethanol-induced narcosis and lethality in ethanol drinking rats. ${ }^{33-35}$ Experimental evidence supports the idea that ACD itself is able to induce addictive like behaviour in rats. Indeed, early studies, have demonstrated that rats self-administer 1 to $5 \%$ (v/v) ACD directly into cerebral ventricles into VTA, ${ }^{28,36}$ and that its intracerebroventricular (icv) infusion induces conditioning-place preference. ${ }^{37,38}$ Rodd-Henricks and coauthors confirmed these data, pointing out that ACD is able to induce a self-administration behaviour directly into VTA, in alcohol-preferring rats. ${ }^{29}$

Several studies highlighted the central effects and role of ACD in the establishment and maintenance of addictive behaviour, even when administered in rats, other than icv. Moreover, systemic ACD injections induce significant stimulus preferences; ${ }^{37}$ intragastric administrations produce conditioning-place preference, ${ }^{39}$ and rats self-inject ACD. ${ }^{40}$ Recently, Cacace and co-authors have investigated the ability of ACD to induce and maintain oral-self administration behaviour in a operant conditioning paradigm, ${ }^{8}$ and the induction of a relapse behaviour upon the introduction of repeated deprivation phases. Furthermore, several studies sustain the role of ACD as a drug of abuse, showing that ACDrats emitted a high number of lever presses during the extinction phase, also that an increase in the lever presses during the reinstatement one, moreover a higher emission of punished responses during the conflict paradigm, ${ }^{22}$ sustaining that ACD itself is able to induce an operant-drinking behaviour.

These experimental evidence, in accordance with previous reports and confirmed by further results, ${ }^{22,41}$ suggests that ACD may be considered as a drug of abuse. Sure enough, in an operant drinking behaviour, rats increase their ACD self-administration intake during reinstatement, following periods of forced abstinence. Furthermore, they increase lever presses for ACD when the reward is delivered together with an aversive stimulus. These studies, taken together, show that ACD is a strong reinforcer, whatever the route of administration is.

\section{Behavioural effects of acetaldehyde}

The impairment in learning and memory and cognitive functions, induced by long-term consumption of $\mathrm{EtOH}$, may be attributed to a neurotoxic injury that induces a chronic degeneration of cholinergic basal forebrain neurons, and hippocampal cholinergic function, comparable to those observed in Alzheimer's disease. ${ }^{42-44}$

ACD is generally considered responsible for the harmful effects of $\mathrm{EtOH}$ occurring through modulation of neurotransmissions pathways in the CNS. ${ }^{4,18,45-47}$ In particular, ACD induces perturbation of cholinergic neurotransmission, ${ }^{48,49}$ a considerable reduction in choline acetyltrasferase (ChAT) expression as a marker of acetylcholine (Ach) expression, in the frontal cortex as well as in the hippocampus and plays a role in the pathogenesis of Alzheimer's disease. ${ }^{18,50,51}$ Jamal and colleagues have analyzed the effects of acute intraperitoneal administration of EtOH in Aldh2 knockout mice, ${ }^{52}$ a model of aldehyde dehydrogenase 2 deficiency in humans, to elucidate the role of ACD in the perturbation of cholinergic function. The authors showed that $\mathrm{EtOH}$ administration was able to induce a decrease in ChAT mRNA and protein levels in aldehyde dehydrogenase 2 knockout mice (Aldh2-KO) but not in wild type mice, suggesting a role for ACD in the mechanism of $\mathrm{EtOH}$ action. Moreover, it has been reported that ACD binds the Apolipoprotein E, a protein directly involved in the alterations of brain morphology and in learning and memory processes, promoting the formation of adducts. ${ }^{53,54}$ In particular, a polymorphism of mitochondrial aldehyde dehydrogenase gene (ALDH2 1/2 polymorphism) may cause the accumulation of ACD which could have a role in Alzheimer's disease, probably due to an altered modulation of the Apolipoprotein E..$^{50,55}$ ACD induces different effects not only on cognitive function, in fact several reports have showed that ACD central administration, or locally formed ACD, is able to increase locomotor activity, ${ }^{56-58}$ while its intraperitoneal injection induces locomotor stimulant effects. ${ }^{19}$ Moreover ACD administration in the hypothalamic arcuate nucleus, a brain area known for its lower presence of ALDH, produces a long lasting induction of locomotion. ${ }^{58}$ Interestingly Escrig and coauthors have found a relationship between anxiogenic effects produced by intraperitoneal high doses of ACD (100 mg/kg) and corticosterone levels, ${ }^{59}$ as marker of endocrine responses, pointing out the role of ACD as mediator of EtOH consumption-induced stress response by ${ }^{60,61}$ These findings are corroborated by previous in vitro studies showing that the inhibition of ALDH by cyanamid produces a significant increase in CRH mRNA in the paraventricular (PVN) and propiomelanocortin (POMC) mRNA in the anterior pituitary, ${ }^{62}$ and that ACD itself is able to induce, in a dose-dependent manner, $\mathrm{CRH}$ release from incubated hypothalamic explants. ${ }^{60}$ These findings can better clarify the implication of ACD in the modulation of central neurotransmitters and peptidergic circuits, contributing to the onset and the maintenance of the emotional and cognitive effects induced by alcohol consumption. Furthermore, in the last year many researches point out to the attention on the role played by the endocannabinoidergic system in the modulation of ethanol-related central effects. Endocannabinoids (ECs) through the activation of the cannabinoid receptor 1 (CB1) broadly localized in the CNS, cause presynaptic inhibition of neurotransmitters release, such as gamma-aminobutyric (GABA), ${ }^{63,64}$ influencing dopamine transmission in the ventral striatum, amygdala and anterior cingulate cortex modulating the motivation approach towards substances of abuse. In particular the antagonist of ECs are able to reduce motivational and reward properties of ACD as mediator of ethanol addiction. ${ }^{22}$

\section{Conclusions}

In the last years, several data commented the possibility that ACD may actually initiate and perpetuate EtOH reinforcement. Furthermore, different researches address the attention to the pivotal role of ACD in the modulation of the central effects of $\mathrm{EtOH}$, since it mediates its consumption, tolerance and reinforcement. ACD might constitute the first hit in EtOH reinforcement, able to establish and maintain addictive behaviour, involving DA transmission in direct and indirect ways. This highlights the relationship and degree of overlap between acetaldehyde's addictive, emotional and cognitive properties. It suggests the real contribution of ACD in central effects of $\mathrm{EtOH}$, driving the studies on ethanol metabolism. This may clarify the elements of individual vulnerability to alcohol addiction to arrange effective strategies aimed to prevent and treat alcohol abuse. 


\section{References}

1. Delker E, Brown Q, Hasin DS. Alcohol consumption in demographic subpopulations: an epidemiologic overview. Alcohol Res 2016;38: 7-15.

2. Beracochea D, Micheau J, Jaffard R. Memory deficits following chronic alcohol consumption in mice: relationships with hippocampal and cortical cholinergic activities. Pharmacol Biochem Behav 1992;42:749-53.

3. Farr SA, Scherrer JF, Banks WA, et al. Chronic ethanol consumption impairs learning and memory after cessation of ethanol. Alcohol Clin Exp Res 2005;29:971-82.

4. Faingold CL, N'Gouemo P, Riaz A. Ethanol and neurotransmitter interactions from molecular to integrative effects. Prog Neurobiol 1998;55:509-35.

5. Prat G, Adan A, Pérez-Pàmies M, Sànchez-Turet M. Neurocognitive effects of alcohol hangover. Addict Behav 2008;33:15-23.

6. Dom G, De Wilde B, Hulstijn W, et al. Decision-making deficits in alcohol-dependent patients with and without comorbid personality disorder. Alcohol Clin Exp Res 2006;30:1670-7.

7. Sullivan EV, Pfefferbaum A. Neurocircuitry in alcoholism: a substrate of disruption and repair. Psychopharmacol 2005;180:583-94.

8. Cacace S, Plescia F, Barberi I, Cannizzaro C. Acetaldehyde oral self administration: evidence from the operant-conflict paradigm. Alcohol Clin Exp Res 2012;36:1278-87.

9. Yonker JE, Nilson LG, Herlitz A, Anthenelli RM. Sex differences in spatial visualization and episodic memory as a function of alcohol consumption. Alcohol Alcohol 2005;40:201-7.

10. Tzambazis K, Stough C. Alcohol impairs speed of information processing and simple choice reaction time and differentially impairs higher-order cognitive abilities. Alcohol Alcohol 2000;35:197-201.

11. Cacace S, Plescia F, Sardo P, Cannizzaro C. Alcohol preference, behavioural reactivity and cognitive functioning in female rats exposed to a three-bottle choice paradigm. Behav Brain Res 2012; 234:11-9.

12. Parsons OA, Nixon SJ. Cognitive functioning in sober social drinkers: a review of the research since 1986. J Stud Alcohol 1998;59:180-90.

13. Cacace S, Plescia F, La Barbera M, Cannizzaro C. Evaluation of chronic alcohol self-administration by a 3-bottle choice paradigm in adult male rats. Effects on behavioural reactivity, spatial learning and reference memory. Behav Brain Res 2011;219:213-20.

14. Agozzino P, Avellone G, Bongiorno D, et al. Determination of the cultivar and aging of Sicilian olive oils using HPLC-MS and linear discriminant analysis. J Mass Spectrom 2010;45:989-95.

15. Sutera FM, De Caro V, Cannizzaro C, et al. Effects of DA-Phen, a dopamine-aminoacidic conjugate, on alcohol intake and forced abstinence. Behav Brain Res 2016; 310:109-18.

16. Zimatkin SM, Liopo AV, Deitrich RA. Distribution and kinetics of ethanol metabolism in rat brain. Alcohol Clin Exp Res 1998;22: 1623-7.

17. Arizzi-LaFrance MN, Correa M, Aragon CMG, Salamone JD. Motor stimulant effects of ethanol injected into the substantia nigra pars reticulata: importance of catalase-mediated metabolism and the role of acetalde- hyde. Neuropsychopharmacol 2006;31:997-1008.

18. Jamal M, Ameno K, Ameno S, et al. Changes in cholinergic function in the frontal cortex and hippocampus of rat exposed to ethanol and acetaldehyde. Neuroscience 2007;144:232-8.

19. Quertemont E, Tambour S, Tirelli E. The role of acetaldehyde in the neurobehavioural effects of ethanol: a comprehensive review of animal studies. Prog Neurobiol 2005;75:247-74.

20. Muggironi G, Fois GR, Diana M. Ethanol-derived acetaldehyde: pleasure and pain of alcohol mechanism of action. Front Behav Neurosci 2013;17:7-87.

21. Peana AT, Muggironi G, Fois GR, et al. Effect of (L)-cysteine on acetaldehyde self-administration. Alcohol 2012;46:489-97.

22. Plescia F, Brancato A, Marino RAM, Cannizzaro C. Acetaldehyde as a drug of abuse: Insight into AM281 administration on operantconflict paradigm in rats. Front Behav Neurosci 2013;7:1-9.

23. Diana M, Peana AT, Sirca D, et al. Crucial role of acetaldehyde in alcohol activation of the mesolimbic dopamine system. Ann NY Acad Sci 2008;1139:307-17.

24. Melis M, Enrico P, Peana AT, Diana M. Acetaldehyde mediates alcohol activation of the mesolimbic dopamine system. Eur J Neurosci 2007;26:2824-33.

25. Deehan GA Jr, Engleman EA, Ding ZM, et al. Microinjections of acetaldehyde or salsolinol into the posterior ventral tegmental area increase dopamine release in the nucleus accumbens shell. Alcohol Clin Exp Res 2013;37:722-9.

26. Brancato A, Plescia F, Marino RAM, et al. Involvement of dopamine D2 receptors in addictive-like behaviour for acetaldehyde. PLoS One 2014;9:e99454.

27. Brown ZM, Amit Z, Smith B. Intraventricular self-administration of acetaldehyde and voluntary con sumption of ethanol in rats. Behav Neural Biol 1980;28:150-5.

28. Brown ZW, Amit Z, Rockman GE. Intraventricular selfadministration of acetaldehyde, but not ethanol, in naive laboratory rats. Psychopharmacol 1979;64:271-6.

29. Rodd-Henricks ZA, Melendeza RI, Zaffaroni A, et al. The reinforcing effects of acetaldehyde in the posterior ventral tegmental area of alcohol-preferring rats. Pharmacol Biochem Behav 2002;72:55-64.

30. Amit Z, Aragon CM. Catalase activity measured in rats naive to ethanol correlates with later voluntary ethanol consumption: possible evidence for a biological marker system of ethanol intake. Psychopharmacol 1988;95:512-5.

31. Aragon CM, Sternklar G, Amit Z. A correlation between voluntary ethanol consumption and brain catalase activity in the rat. Alcohol 1985;2:353-6.

32. Hamby-Mason R, Chen JJ, Schenker S, et al. Catalase mediates acetaldehyde forma- tion from ethanol in fetal and neonatal rat brain. Alcohol Clin Exp Res 1997;21:1063-72.

33. Tampier L, Quintanilla ME, Mardones J. Influence of 3-amino1,2,4-triazole pretreatment on ethanol induced narcosis in rats. IRCS Med Sci 1979;7:390.

34. Tampier L, Mardones J. Effect of administration order of aminotriazole and ethanol on brain catalase activity and ethanol induced nacosis timenin UChA and UChB rats. IRCS Med Sci 1983;11:468.

35. Tampier L, Quintanilla ME, Mardones J. Effect of 3-amino-1,2,4-triazole on narcosis time and lethality of ethanol in UChA rats. Alcohol 1988;5:5-8.

36. McBride WJ, Li TK, Deitrich RA et al. Involvement of acetaldehyde in alcohol addiction. Alcohol Clin Exp Res 2002;26:114-9.

37. Quertemont E, De Witte P. Conditioned stimulus preference after acetaldehyde but not ethanol injections. Pharmacol Biochem Behav 2001;68:449-54.

38. Smith BR, Amit Z, Splawinsky J. Conditioned place preference induced by intraventricular infusions of acetaldehyde. Alcohol 1984;1:193-5.

39. Peana AT, Assaretti AR, Muggironi G, et al. Reduction of ethanolderived acetaldehyde induced motivational properties by L-cysteine. Alcohol Clin Exp Res 2009;33:43-8.

40. Myers W, Ng K, Singer G. Ethanol preference in rats with a prior history of acetaldehyde self-administration. Experientia 1984;40: 1008-10.

41. Peana AT, Muggironi G, Diana M. Acetaldehyde reinforcing effects: 
a study on oral self-administration behaviour. Front Psychiatry 2010;1:23.

42. Arendt Th, Bigl V, Arendt A, Tennstedt A. Loss of neurons in the nucleus basalis of Meynert in Alzheimer's disease, paralysis agitans and Korsakoff's disease. Acta Neuropath 1983;61:101-8.

43. Beracochea D, Lescaudron L, Verna A, Jaffard R. Neuroanatomical effects of chronic ethanol consumption on dorsomedial and anterior thalamic nuclei and on substantia innominata in mice. Neurosci Lett 1987;73:81-4.

44. White AM, Swartzwelder HS. Hippocampal function during adolescence: a unique target of ethanol effects. Ann NY Acad Sci 2004;1021:206-20.

45. Foddai M, Dosia G, Spiga S, Diana M. Acetaldehyde increases dopaminergic neuronal activity in the VTA. Neuropsychopharmacol 2004;29:530-6.

46. Han QP, Dryhurst G. Influence of glutathione on the oxidation of 1methyl-6-hydroxy-1,2,3,4-tetrahydro-beta-carboline: chemistry of potential relevance to addictive and neurodegenerative consequences of ethanol use. J Med Chem 1996;39:1494-508.

47. Zuddas A, Corsini GU, Schinelli S, et al. Acetaldehyde directly enhances MPP+ neurotoxicity and delays its elimination from the striatum. Brit Res 1989;501:11-22.

48. Kuriyama K, Ohkuma S, Tomono S, Hirouchi M. Effects of alcohol and acetaldehyde on metabolism and function of neurotransmitter systems in cerebral cortical neurons in primary culture. Alcohol 1987; 1:685-9.

49. Vakili A, Tayebi K, Jafari MR, et al. Effect of ethanol on morphine state-dependent learning in the mouse: involvement of GABAergic, opioidergic and cholinergic systems. Alcohol 2004;39:427-32.

50. Wang B, Wang J, Zhou S, et al. The association of mitochondrial aldehyde dehydrogenase gene (ALDH2) polymorphism with susceptibility to late-onset Alzheimer's disease in Chinese. J Neurol Sci 2008;268:172-5.

51. Self RL, Smith KJ, Mulholland PJ, Prendergast MA. Ethanol exposure and withdrawal sensitizes the rat hippocampal CA1 pyramidal cell region to beta-amyloid (25-35)-induced cytotoxicity: NMDA receptor involvement. Alcohol Clin Exp Res 2005;29:2063-9.

52. Jamal M, Ameno K, Ruby M, et al. Ethanol- and acetaldehydeinduced cholinergic imbalance in the hippocampus of Aldh2-knockout mice does not affect nerve growth factor or brain-derived neurotrophic factor. Brain Res 2013;1539:41-7.
53. Plassman BL, Welsh-Bohmer KA, Bigler ED et al. Apolipoprotein E epsilon 4 allele and hippocampal volume in twins with normal cognition. Neurology 1997;48:985-9.

54. Greenwood PM, Sunderland T, Friz JL, Parasuraman R. Genetics and visual attention: selective deficits in healthy adult carriers of the epsilon 4 allele of the apolipoprotein E gene. P Natl Acad Sci USA 2000;97:11661-6.

55. Jamal M, Ameno K, Miki T, et al. High ethanol and acetaldehyde impair spatial memory in mouse models: opposite effects of aldehyde dehydrogenase 2 and apolipoprotein E on memory. Pharmacol Biochem Behav 2012;101:443-9.

56. Correa M, Arizzi MN, Betz A, et al. Open field locomotor effects in rats after intraventricular injections of ethanol and the ethanol metabolites acetaldehyde and acetate. Brain Res Bull 2003;62:197-202.

57. Correa M, Manrique HM, Font L, et al. Reduction in the anxiolytic effects of ethanol by centrally formed acetaldehyde: the role of catalase inhibitors and acetaldehyde-sequestring agents. Psychopharmacol 2008;200:455-64.

58. Correa M, Arizzi-LaFrance MN, Salamone JD. Infusions of acetaldehyde into the arcuate nucleus of the hypothalamus induce motor activity in rats. Life Sci 2009;84:321-7.

59. Escrig MA, Pardo M, Aragon CM, Correa M. Anxiogenic and stressinducing effects of peripherally administered acetaldehyde in mice: similarities with the disulfiram-ethanol reaction. Pharmacol Biochem Behav 2012;100:404-12.

60. Cannizzaro C, La Barbera M, Plescia F, et al. Ethanol modulates corticotropin releasing hormone release from the rat hypothalamus: does acetaldehyde play a role? Alcohol Clin Exp Res 2010;34:588-93.

61. Cannizzaro C, Plescia F, Cacace S. Role of acetaldehyde in alcohol addiction: current evidence and future perspectives. Malta Med J 2011;23:27.

62. Kinoshita H, Jessop DS, Finn DP, et al. Acetaldehyde, a metabolite of ethanol, activates the hypothalamic-pituitary-adrenal axis in the rat. Alcohol 2001;36:59-64.

63. Brancato A, Lavanco G, Cavallaro A, Plescia F, Cannizzaro C. The use of the emotional-object recognition as an assay to assess learning and memory associated to an aversive stimulus in rodents. $\mathrm{J}$ Neurosci Methods 2016;274:106-15.

64. Cavallaro A, Martines F, Cannizzaro C, et al. Role of cannabinoids in the treatment of tinnitus. Acta Medica Mediterranea 2016; $32: 463$. 